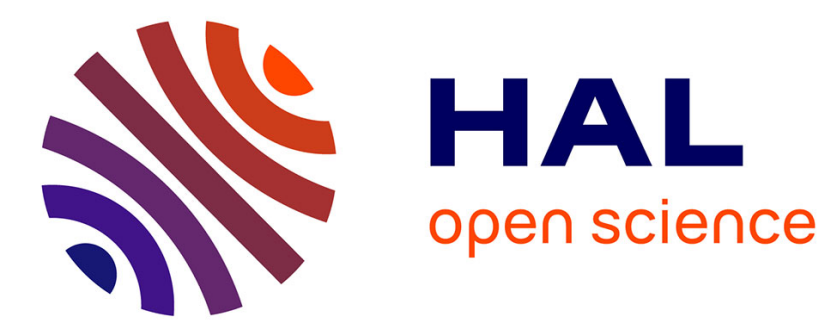

\title{
Laser-heated microswimmers: optical manipulation of active particles
}

\author{
Aloïs Würger
}

\section{To cite this version:}

Aloïs Würger. Laser-heated microswimmers: optical manipulation of active particles. Optical Manipulation and Structured Materials Conference 2020, Apr 2020, Yokohama, Japan. 10.1117/12.2573516 . hal-02876019

\section{HAL Id: hal-02876019 https://hal.science/hal-02876019}

Submitted on 20 Jun 2020

HAL is a multi-disciplinary open access archive for the deposit and dissemination of scientific research documents, whether they are published or not. The documents may come from teaching and research institutions in France or abroad, or from public or private research centers.
L'archive ouverte pluridisciplinaire HAL, est destinée au dépôt et à la diffusion de documents scientifiques de niveau recherche, publiés ou non, émanant des établissements d'enseignement et de recherche français ou étrangers, des laboratoires publics ou privés. 


\title{
Laser-heated microswimmers: optical manipulation of active particles*
}

\author{
Alois Würger \\ Laboratoire Ondes et Matière d'Aquitaine, Université de Bordeaux 8 CNRS, 33405 Talence, France
}

\begin{abstract}
In recent years various experiments reported translational and rotational motion of laser-heated Janus colloids, including steering through feedback and collective effects. We discuss optical actuation and swimming mechanisms, in terms of the underlying thermal surface forces and slip velocities, the latter providing hydrodynamic boundary conditions.
\end{abstract}

Keywords: active matter, optical manipulation, thermophoresis, diffusiophoresis, surface forces

\section{INTRODUCTION}

Laser-heated metal or carbon nanostructures provide versatile local heat sources, with applications ranging from photothermal imaging [1] to thermally powered artificial microswimmers [2]. The latter rely on the conversion of heat tomotion; experimental realizations include Janus colloids driven by surface forces [3], and interfacial particles that are advected by their self-generated Marangoni flow $[4,5]$. Force-free localization and steering have been achieved by temporal [6] or spatial [7] modulation of the laser power. Cluster formation and collective properties of active colloids have been discussed in terms of the particle geometry [8] and hydrodynamic interactions [9].

In contrast, in recent years a variety of heat-flow induced swimming mechanisms have been pointed out, such as diffusiophoresis due to local phase separation in a near-critical mixture [10] or thermo-osmotic flow towards
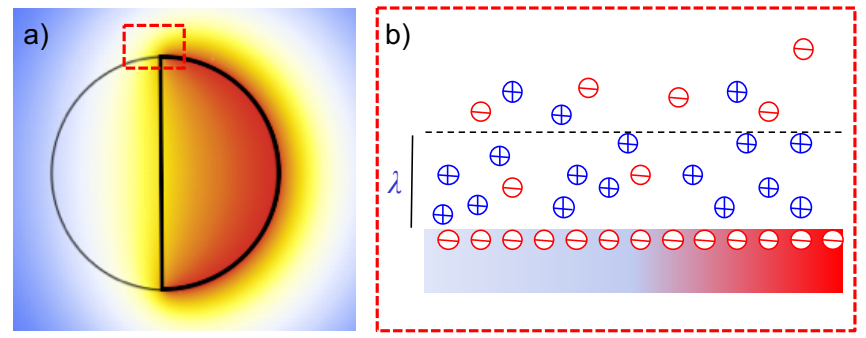

FIG. 1: a) Temperature map of a laser-heated Janus particle. The temperature is higher at the

light-absorbing gold or carbon cap, resulting in a parallel component $\nabla_{\|} T$ of the temperature gradient along the surface. b) Active motion arises from the forces exerted by the particle on the liquid, mostly related either to the electric-double layer (shown here) or to the van der Waals interactions. The interaction length $\lambda$ is of the order of nanometers, much smaller than the particle size.

\footnotetext{
*alois.wurger@u-bordeaux.fr; phone +33(0)5 40006202
}

a hot spot in a capillary [11]. These experiments also revealed strong dependencies on material properties, such as the reverse swimming direction upon adding a nonionic surfactant [3] or an increase of the thermo-osmotic velocity due to a copolymer coating [11]. Specific-ion effects were reported for silica colloids carrying a gold cap: their swimming velocity in a $10 \mathrm{mM} \mathrm{NaCl}$ solution changed significantly when replacing the cation with Lithium, or the anion with hydroxide [12].

Active motion arises from the creep flow along the particle surface. Its axisymmetric component results in linear motion of the Janus particle, as observed in the above mentioned experiments. In various instances, however, active particles show also rotational motion. Thus Janus colloids with a metal cap of irregular shape move along complex trajectories [4, 13-15].

Here we discuss main mechanism for self-propulsion of laser-heated particles and typical settings resulting in an active particle rotation.

\section{SELF-PROPULSION MECHANISMS}

Driving active colloids wtih thermal or chemical gradients, or an AC electric field, relies on osmosis: The applied field induces a non-equilibrium temperature or solute concentrations in the interaction layer. The resulting diffusion of heat or ions drags the liquid along the surface, which by reaction causes the particle to move in the opposite direction.

Thermo-osmosis, that is fluid flow due to a temperature gradient, was first reported and rationalized by Derjaguin [16]. A microsopic observation was achieved recently through tracking of tracer particles [11] in a micron-size capillary. Regarding the electric double layer shown in Fig. 1b, the charged liquid is dragged towards higher temperature, and the Janus particle moves to the left.

When adding a small amount of salt, however, the electrolyte Seebeck effect turns out to be the dominant driving mechanism: Because of their specific solvation enthalpies, cations and anions accumulate at hot or cold surfaces, as shown schematically in Fig. 2a. For micron size particles, this thermocharges may attain values of about 100 elementary charges [17]. Then the parti- 

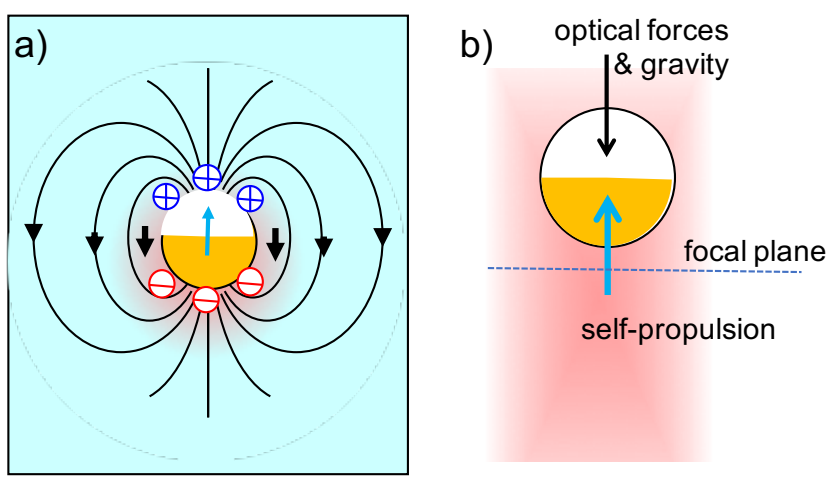

FIG. 2: a) Thermoelectric driving mechanism. In a salt solution, the cations and anions diffuse along the temperature gradient, albeit at different pace. As a consequence, one species accumulates at the hot side, and the other one at the opposite one. These positive and negative thermocharges induce a dipolar electric field, as shown by the black lines, and the Janus particle moves due to electrophoresis in this thermoelectric field. b) Eperimental setup for measuring self-propulsion used in Ref. [12]. A gold capped silica particle is trapped in a focussed laser beam; gravity and radiation pressure would result in a stationary position bell below the focal plane. Yet the upward self-propulsion overcomes this forces, resulting in a vertical position above the focal plane. Note that, because of the heavy gold cap, the particle axis always points upwards.

cle moves due to electrophoresis in this self-engendered electric dipole field $[18,19]$. Such an ion-specific effect was reported for gold-capped silica particles heated by in a focussed laser beam. As shown schematically in Fig. $2 \mathrm{~b}$, despite the downward radiation pressure and gravity, the particles moved above the focal plane, and its height depended on the salt $\mathrm{NaCl}, \mathrm{LiCl}, \mathrm{NaOH}[12]$. These experimental findings also confirm previous observations on passive particles in an external temperature gradient [20$22]$.

A very efficient driving mechanism occurs in nearcritical water-oil mixtures at a temperature just below a lower critical solution point [10]. Heating the particle just above $T_{c}$, induces phase separation in a small volume around the cap, as shown in Fig. 3. The outer boundary of this droplet is at the critical temperature $T_{c}$. Its composition depends on the wetting properties of the surface: The left panel shows an overall hydrophilic particle, which favors the water-rich phase where darker blue indicates higher water content [24]. In the right panel, the light-absorbing cap (red) is hydrophobic and adsorbs oil (yellow), whereas the passive hemisphere (white) is hydrophilic. As a consequence, the critical droplet comprises two compartments of opposite excess composition.

Within the range of van der Waals forces, the particle surface attracts more water (or oil) than corresponding to

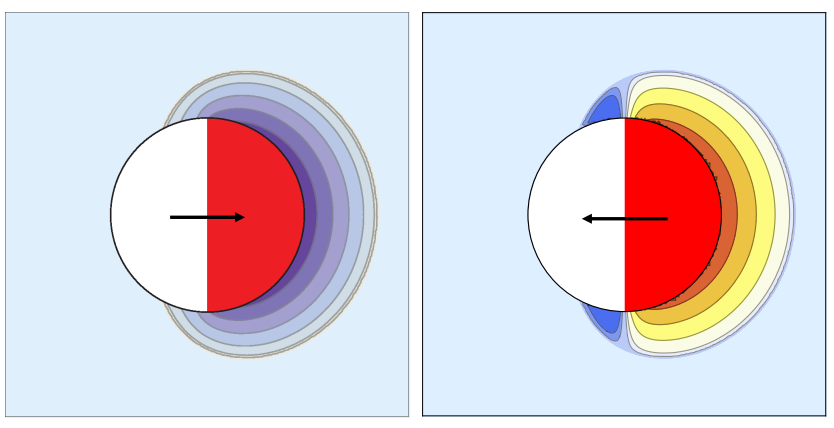

FIG. 3: Diffusiophoresis in a near-critical binary mixture at the critical composition and just below the lower critical solution temperature $T_{c}$. In the vicinity of the heated cap (red), $T>T_{c}$ induces a water-rich phase

(dark blue) at hydrophilic surfaces, and an oil-rich

phase (yellow) close to hydrophobic surfaces. In two cases shown, the composition gradient points to the left or to the right, resulting in opposite motion of the Janus particle.

the equilibrium composition. This excess drives a creep flow and results in self-diffusiophoresis of the Janus colloid, at a velocity which exceeds that of thermo-osmosis discussed above. Since the water concentration at the surface increases towards higher temperature in the left panel, and towards lower $T$ at the right, the particle moves in opposite directions, as indicated by the arrows [24]. A variety of phenomena have been observed for such laser-powered diffusiophoretic swimmers [7, 10, 15, 25].

\section{GUIDING PARTICLES ALONG AN INTENSITY GRADIENT}

The thermodynamic forces acting in the interaction layer shown in Figs. 1-3, induce a creep flow along the particle surface and, by reaction, in a rectilinear motion of the Janus colloid along its axis. The particle moves the hot hemisphere at the front or at the rear, depending on the detail of the surface forces. In the case of a focussed laser beam, its intensity $I(\mathbf{r})$ may significantly vary over the particle surface. As a consequence of the non-uniform absorption, the surface temperature is not axisymmetric. Then the surface forces exert a torque on the particle axis n [23],

$$
\mathbf{T}=\left(\mu-\mu^{\prime}\right) \mathbf{n} \times \nabla I,
$$

where the parameters $\mu$ and $\mu^{\prime}$ account for the surface properties on the two hemispheres. This torque aligns the axis $\mathbf{n}$ on the intenstity gradient $\nabla I$. With an appropriate choice of the surface parameters, the active particle always moves towards the beam center where the intensity is maximum, as illustrated in Fig. 4.

Such a steering effect was indeed observed for Janus particles in a near-critical water-lutidine mixture: In a 
a)

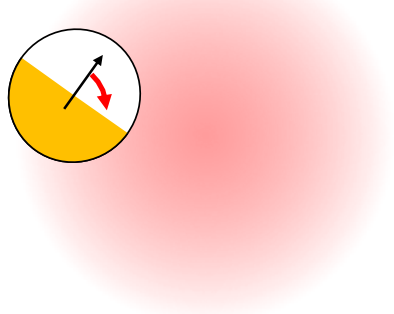

b)

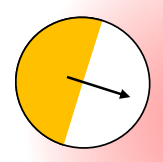

FIG. 4: a) Active particle in a Gaussian laser beam. The torque (1) turns the particle axis $\mathbf{n}$ towards the beam center. b) The stable orientation is achieved if $\mathbf{n}$ is aligned on the intensity gradient $\nabla I$. Moving the beam, it is then possible to guide the particle along a given trajectory. On the other hand,

capillary of with a linearly increasing laser power, $I(z)=$ $[1-\kappa|z|) I_{\max }$, the particles move along the $z$-axis towards the position of maximum power, $z=0$, where they get trapped, in accordance with Eq. (1) [25].

\section{ROTATION DUE TO INHOMOGENEOUS SURFACES}

The torque (1) arises from the superposition of the particle's linear motion with an applied gradient. It does not result in a stationary rotational state but rather in polarization along the gradient. A permanent rotation may occur, however, for colloidal spheres with non-uniform surface properties, where the angular velocity is given by the surface integral

$$
\Omega=-\frac{3}{8 \pi a^{3}} \oint d \mathbf{S} \times \mathbf{v}_{s}
$$

with the slip velocity $\mathbf{v}_{s}$. For Janus particles with irregular shapes of the active cap, complex 3D trajectories have been observed which result from the superposition of linear and rotational motion [13]. A similar behavior was observed for particles trapped at a fluid interface [14]; due to contact line pinning, the angular velocity is perpendicular to the interface, resulting in epicycloid like trajectories.

Rotational motion also occur for active particles that are trapped by a tweezer potential. Circular orbits have been observed for gold colloids in a near-critical binary mixture [15] and for interfacial polystyrene particles with iron oxide inclusions [4]. Their origin is not clear at present, it could be related to non-spherical shape.

The author acknowledges support from the French National Research Agency through contracts ANR-13-IS040003 and ANR-19-CE30-0012-01, and from ERC through contract 772725 .
[1] D. Boyer, P. Tamarat, A. Maali, B. Lounis, and M. Orrit, Science 297, 1160 (2002).

[2] C. Bechinger, R. Di Leonardo, H. Lwen, C. Reichhardt, Giorgio Volpe, and Giovanni Volpe, Rep. Mod. Phys. 88, 045006 (2016)

[3] H.-R. Jiang, N. Yoshinaga, M. Sano, Phys. Rev. Lett. 105, $268302(2010)$

[4] A. Girot, N. Danné, A. Würger, T. Bickel, F. Ren, J. C. Loudet, and B. Pouligny, Langmuir 32, 2687 (2016).

[5] A. Würger, J. Fluid Mech. 752, 589 (2014).

[6] A. P. Bregulla, H. Yang, and F. Cichos, ACS Nano 8, $6542(2014)$.

[7] C. Lozano, B. ten Hagen, H. Löwen, and C. Bechinger, Nat. Commun. 7, 12828 (2016)

[8] I. Buttinoni, J. Bialke, F. Kümmel, H. Löwen, C. Bechinger, and T. Speck, Phys. Rev. Lett. 110, 238301 (2013).

[9] A. Zöttl and H. Stark, Phys. Rev. Lett. 112, 118101 (2014).

[10] I. Buttinoni, G. Volpe, F. Kümmel, G. Volpe, and C. Bechinger, J. Phys.: Cond. Mat. 24, 284129 (2012).

[11] A. P. Bregulla, A. Würger, K. Günther, M. Mertig, and F. Cichos, Phys. Rev. Lett. 116, 188303 (2016).

[12] S. Simoncelli, J. Summer, S. Nedev, P. Kühler, and J. Feldmann, Small 29, 2854 (2016).

[13] R.J. Archer, A.I. Campbell and S.J. Ebbens, Soft Matter
11, 6872 (2015).

[14] X. Wang, M. In, C. Blanc, A. Würger, M. Nobili, A. Stocco, Langmuir 33, 13766 (2017).

[15] F. Schmidt, A. Magazzù, A. Callegari, L. Biancofiore, F. Cichos, and G. Volpe, Phys. Rev. Lett. 120, 068004 (2018).

[16] B.V. Derjaguin, G.P. Sidorenkov, Doklady Akad. Nauk. SSSR,32, 622 (1941).

[17] A. Majee and A. Würger, Phys. Rev. Lett. 108, 11803 (2012).

[18] A. Würger, Rep. Prog. Phys. 73, 126601 (2010).

[19] A. Ly, A. Majee and A. Würger, New J. Phys. 20, 025001 (2018).

[20] S. A. Putnam and D. G. Cahill, Langmuir 21, 5317 (2005).

[21] D. Vigolo, S. Buzzaccaro, and R. Piazza, Langmuir 26, 7792 (2010).

[22] K. A. Eslahian, A. Majee, M. Maskos, A. Würger, Soft Matter 10, 1931 (2014).

[23] T. Bickel, G. Zecua, and A. Würger, Phys. Rev. E 89, 050303 (2014)

[24] A. Würger, Phys. Rev. Lett. . 115, 188304 (2015).

[25] C. Lozano and Clemens Bechinger, Nature Comm. 10, 2495 (2019) 